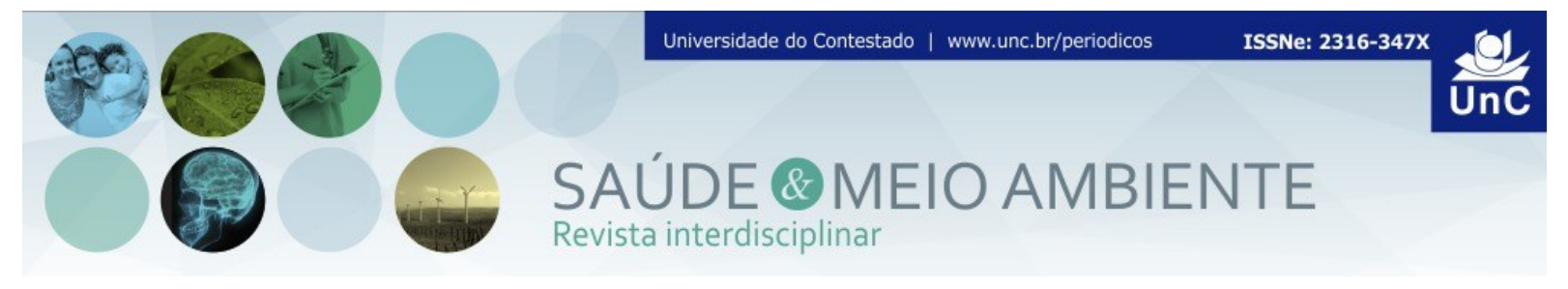

\title{
AUTO PERCEPÇÃO DA QUALIDADE DE VIDA EM ESCOLARES ADOLESCENTES
}

\author{
João Carlos Raiser ${ }^{1}$ \\ Chelin Auswaldt Steclan ${ }^{2}$
}

\begin{abstract}
RESUMO
A auto percepção sobre a Qualidade de vida é definida pela sua relação e posicionamento com a vida, sendo de suma importância o conhecimento desta variável para o planejamento e execução de ações que promovam a melhoria da qualidade de vida das pessoas. Esta forma de qualificação é estudada em diversas populações de diferentes faixas etárias, sendo aqui especificamente em adolescentes escolares, os quais constituirão a população futura ativa. Tendo isso, o presente trabalho teve como objetivo caracterizar a auto percepção da qualidade de vida de escolares do sétimo ao nono ano, comparando instituições localizadas em perímetro urbano e rural. Achou-se para este estudo que grande parte dos adolescentes possuem auto percepção do estado de saúde como bom a ótimo, contudo, os escolares do perímetro rural relatam que problemas, como o sentimento de dor, atrapalha atividades físicas do dia a dia. Problemas de ordem emocional foram desconsiderados como fatores interventores pela população estudada. Análises populacionais, mesmo que de auto percepção, são ótimos indicadores sociais, e de saúde nos dias atuais, pois proporcionam a caracterização dos mesmos, possibilitando ações prospectivas positivas.
\end{abstract}

Palavras Chave: Qualidade de vida. Adolescentes. Auto percepção. Prospecção futura.

\section{SELF-PERCEPTION OF QUALITY OF LIFE AMONG ADOLESCENT STUDENTS}

\begin{abstract}
Self-perception about Quality of life is defined by its relationship and positioning with life, being extremely important the knowledge of this variable for the planning and execution of actions that promote the improvement of people's quality of life. This form of qualification is studied in diverse populations of different age groups, being

\footnotetext{
${ }^{1}$ Acadêmico de Enfermagem, Curso de Enfermagem, Universidade do Contestado, cidade de Mafra, Santa Catarina. Brasil. E-mail: joaoraiser2011@hotmail.com

${ }^{2}$ Bióloga e Doutora em Biologia Celular e Molecular, Professora titular da Universidade do Contestado, cidade de Mafra, Santa Catarina. Brasil. E-mail: chelin@unc.br
} 
specifically here in school adolescents, who will constitute the future active population. The aim of this study was to characterize the self - perception of the quality of life of schoolchildren from the seventh to the ninth grade, comparing institutions located in urban and rural perimeters. It was found for this study that a great part of the adolescents has self-perception of the state of health as good to great, however, the students of the rural perimeter report that problems, like the feeling of pain, disrupt physical activities of the day to day. Emotional problems were disregarded as intervening factors by the study population. Population analyzes, even of self-perception, are excellent social indicators, and health in the present day, since they provide the characterization of the same, enabling positive prospective actions.

Keywords: Life Quality. Adolescents. Self-perception. Future prospecting.

\section{INTRODUÇÃO}

A organização mundial da saúde (OMS) define "qualidade de vida" como: "a percepção do indivíduo sobre a sua posição na vida, no contexto da cultura e dos sistemas de valores nos quais ele vive, e em relação a seus objetivos, expectativas, padrões e preocupações" (The WHOQOL Group, 1995).

O perfil da qualidade de vida dos escolares atualmente tem despertado grande interesse para a comunidade científica e organizações públicas/privadas, principalmente por se tratar da futura geração que entrará no mercado de trabalho e usufruirá dos serviços prestados, por exemplo, de educação e saúde (VINGILIS et al., 2002; QUINTAS, 2004; KUNKEL; OLIVEIRA; PERES, 2009; RAYMUNDO; KUHNEN, 2010). E para retratar a qualidade de vida deste público especifico, temse voltado os objetivos e perspectivas para os ambientes educativos, onde há o direcionamento para o individuo e/ou grupo social, que se resume principalmente nas comunidades escolares, pois são riquíssimas fontes de formação, produção e disseminação de conhecimento.

Ações pedagógicas de ensino e conscientização sobre qualidade de vida tem sido amplamente explorada por diversas comunidades científicas e do saber (QUINTAS, 2004; RAYMUNDO; KUHNEN, 2010), os quais relatam, por exemplo, a utilização de ambientes escolares de ensino como grandes e melhores propagadores da conscientização e promoção da boa qualidade e estilo de vida.

A população de adolescentes é um potencial alvo para avaliações de qualidade vida, visto que é o período da vida em que a saúde está no seu melhor estado e com tempo hábil para a execução de atividades físicas e sociais, além de que nesta fase fatores como hábitos (ex. alimentar e estilo de vida) e vícios (ex. tabaco e álcool) tendem-se a consolidar e arrastarem-se para a fase adulta, trazendo danos muitas vezes irreversíveis ao estilo e qualidade de vida (KUNKEL; OLIVEIRA; PERES, 2009; VINGILIS et al., 2002). 
De fato, através de avaliação de projetos de promoção à saúde, constata-se que a qualidade e estilo de vida de jovens é alterada por evidências prospectivas, e há realmente mudanças em relação ao estilo de vida dos que passaram a ter uma visão positiva de saúde por intervenções educativas (BARBOSA FILHO et al., 2014).

Contudo, o estudo da qualidade de vida em diversas populações é feito principalmente através da aplicação de questionários que contemplam as abordagens e ações referentes aos hábitos e ações do indivíduo. Sendo assim, este trabalho teve como objetivo avaliar a qualidade de vida de adolescentes escolares através da aplicação de questionário previamente validado, buscando principalmente estudar as variações em relação a públicos distintos.

\section{METODOLOGIA}

Teve-se como método adotado para a pesquisa a coleta de dados através da aplicação de questionário com perguntas fechadas e de múltipla escolha, adaptadas ao público alvo (escolares). Gil (1999) defende que questionários como técnicas de investigação compostos por questões apresentadas por escrito às pessoas tem 0 objetivo de conhecer suas opiniões, crenças, sentimentos, interesses, expectativas, e situações vivenciadas, o que de fato é esperado na proposta em questão.

Para avaliação da qualidade de vida foi utilizado o questionário SF - 36, previamente validado (CICONELLI et al., 1999), o qual trata-se de um questionário que retrata a opinião do entrevistado sobre sua saúde, a forma como ele se sente, e a capacidade de desempenhar as atividades do dia a dia.

Os questionários foram aplicados em duas instituições de ensino regular do município de Mafra, estado de Santa Catarina - Brasil, sendo uma localizada no perímetro urbano e outro no perímetro rural. As instituições participantes declararam aceitar da pesquisa, e após isto foram eleitas três turmas de cada instituição receptora da proposta, sendo elas do sétimo, oitavo e nono ano, num total de 146 participantes. Antes de realizar a coleta de dados, os alunos foram informados da pesquisa e aqueles que mostraram interesse em participar levaram para casa um termo de livre esclarecimento e consentimento de participação que deveria ser assinada pelos pais, ou responsável legal, e teria de ser entregue na hora da entrevista junto ao questionário.

Em dias aleatórios, com agendamento prévio, o questionário foi aplicado sob a supervisão dos pesquisadores do trabalho, sendo cada pergunta e alternativas lidas pausadamente aos entrevistados e esclarecidas em caso de dúvidas. Os alunos participantes responderam e entregaram os questionários logo após o término da aplicação, e junto ao termo de consentimento esclarecido.

A análise dos dados foi realizada conforme Kenlinger (1980), onde foram categorizados, ordenados, e sumarizados para que assim as entrevistas sejam transformadas para melhor interpretação. O tratamento dos dados foi realizado por 
meio da descrição da amostra com o auxílio de programa estatístico para a confecção dos gráficos e tabelas, podendo assim facilitar a interpretação de determinadas variáveis identificadas pela equipe de trabalho, assim como, hipóteses apontadas durante o estudo. O pacote estatístico utilizado foi o formato Demo do programa GraphPad Prism 6.0 disponibilizados na página eletrônica do programa.

\section{RESULTADOS E DISCUSSÃO}

O perfil dos escolares, independente da faixa etária, tem sido vastamente estudado visando que esta população é a prospecção da população futura. Problemas quanto a qualidade de vida, assim como estilo de vida, são um dos mais debatidos e trabalhados (CAVALCANTE et al., 2008). Em estudo sobre qualidade de vida com adolescentes escolares houve a identificação de 3 principais fatores que levam a sua classificação como grupo de risco, sendo eles: baixa rotina de atividade física, baixo consumo de verduras e legumes, e a não utilização de preservativos (FARIAS JUNIOR et al., 2008). Tem-se descrito também, que a baixa atividade física é positivada pela presença de fatores e comportamentos que levam ao sedentarismo (TENÓRIO et al., 2010). Para estes, assim como para outros estudos conclui-se a carência de programas de promoção de saúde para este público em específico como forma de melhoria e reversão de quadros mais agravados.

Os questionários foram aplicados para alunos de sétimo a nono ano do ensino regular, não sendo realizada a identificação dos mesmos. Os questionários respondidos foram coletados junto às declarações de consentimento assinada pelos pais dos participantes. Após reunião dos documentos, os mesmos foram compilados e comparados frente às duas instituições participantes. De primeira ordem, vale ressaltar que não houve diferenças quanto aos padrões de resposta, contudo, houve discrepâncias muitas vezes na porcentagem das mesmas. Em geral, os adolescentes classificaram sua saúde entre excelente a boa, a qual no último ano se manteve na mesma situação a muito melhor (Gráficos 1 e 2, respectivamente).

Gráfico 1 - Perfil das respostas para a pergunta 1, sendo A escolares da área rural, e B escolares da área urbana. No eixo ' $x$ ' apresenta-se as alternativas.
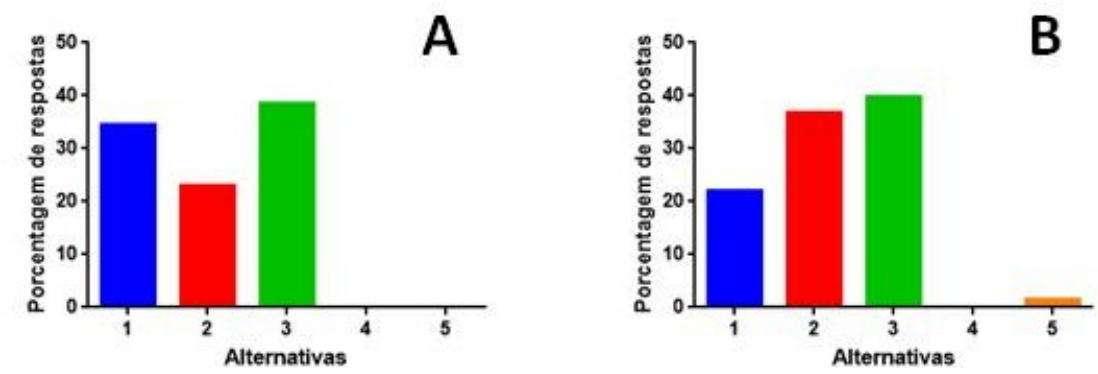

Saúde Meio Ambient. v. 6, n. 1, p. 43-53, jan./jun. 2017 
Gráfico 2 - Perfil das respostas para a pergunta 2, sendo A escolares da área rural, e B escolares da área urbana. No eixo ' $x$ ' apresenta-se as alternativas.
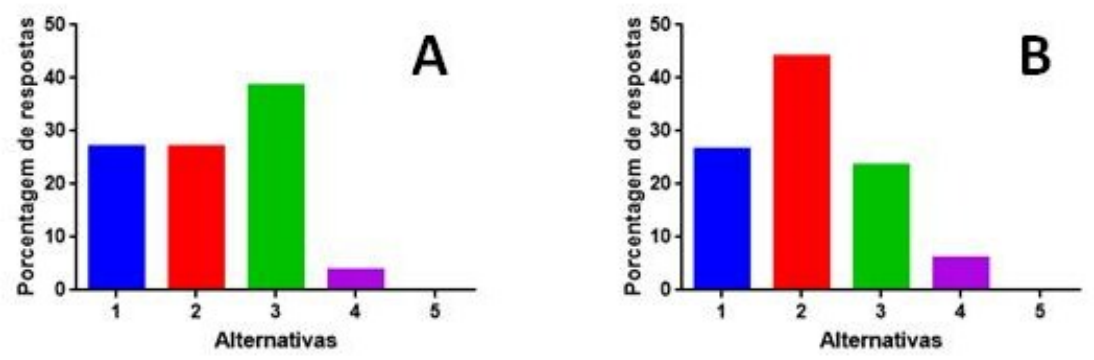

A capacidade de realizar atividades em um dia comum foi questionada em relação a vários fatores, e achou-se que os escolares da área rural de caracterizam sentir muita ou alguma dificuldade em realizar atividades desde rotineiras como tomar banho e se vestir à atividades rigorosas como correr e participar de esportes árduos. Já para os escolares da área urbana, achou-se o inverso para a mesma avaliação, sendo que para as mesmas atividades houve consenso para ausência de dificuldades para realização das atividades (tabela 1).

Tabela 1 - Dados compilados da questão 3, dividido por alternativas $(\mathrm{a}-\mathrm{j})$ e áreas de estudo (rural e urbana). Os valores sublinhados são aqueles de referência para os resultados discutidos.

\begin{tabular}{|c|c|c|c|c|c|c|}
\hline & \multicolumn{3}{|c|}{ Área rural } & \multicolumn{3}{|c|}{ Área urbana } \\
\hline & $\begin{array}{l}\text { Sim, dificulta } \\
\text { muito }\end{array}$ & $\begin{array}{l}\text { Sim, dificulta } \\
\text { um pouco }\end{array}$ & $\begin{array}{c}\text { Não, não } \\
\text { dificulta de } \\
\text { modo algum }\end{array}$ & $\begin{array}{l}\text { Sim, dificulta } \\
\text { muito }\end{array}$ & $\begin{array}{l}\text { Sim, dificulta } \\
\text { um pouco }\end{array}$ & $\begin{array}{c}\text { Não, não } \\
\text { dificulta de } \\
\text { modo algum }\end{array}$ \\
\hline$a$ & $\underline{46,2}$ & 30,8 & 23,1 & 1,5 & 38,2 & $\underline{60,3}$ \\
\hline $\mathbf{b}$ & 30,8 & 30,8 & $\underline{34,6}$ & 0,0 & 5,9 & $\underline{45,6}$ \\
\hline c & $\underline{34,6}$ & 30,8 & 30,8 & 0,0 & 2,9 & $\underline{11,8}$ \\
\hline d & $\underline{38,5}$ & 23,1 & 38,5 & 7,4 & 16,2 & $\underline{38,2}$ \\
\hline $\mathbf{e}$ & $\underline{50,0}$ & 19,2 & 26,9 & 2,9 & 11,8 & $\underline{42,6}$ \\
\hline$f$ & $\underline{42,3}$ & 19,2 & 30,8 & 2,9 & 10,3 & $\underline{44,1}$ \\
\hline g & 26,9 & $\underline{38,5}$ & 23,1 & 2,9 & 16,2 & 39,7 \\
\hline $\mathrm{h}$ & 26,9 & $\underline{46,2}$ & 19,2 & 5,9 & 17,6 & $\underline{36,8}$ \\
\hline $\mathbf{i}$ & $\underline{38,5}$ & 30,8 & 15,4 & 4,4 & 11,8 & $\underline{42,6}$ \\
\hline $\mathbf{j}$ & $\underline{61,5}$ & 0,0 & 30,8 & 4,4 & 0,0 & 44,1 \\
\hline
\end{tabular}

O estado da saúde física dos participantes também foi avaliado quanto a atividades de trabalho ou atividades regulares do dia a dia, e para este tópico foi encontrada apenas diferença quando a presença ou não de dificuldades para execução de tarefas entre as duas populações do estudo. No resultado encontrado, os escolares da área rural relataram que o estado de saúde atrapalhou, de alguma forma, nas últimas 4 semanas, as atividades do trabalho ou regulares, provavelmente devido as tarefas domiciliares que o ambiente rural concilia. Já para os outros tópicos, o estado da saúde não foi um fator determinante para a execução de atividades (Tabela 2 ). 
Tabela 2 - Dados compilados da questão 4, dividido por alternativas $(a-d)$ e áreas de estudo (rural e urbana). Os valores sublinhados são aqueles de referência para os resultados discutidos.

\begin{tabular}{|c|c|c|c|c|}
\cline { 2 - 5 } \multicolumn{1}{c|}{} & \multicolumn{2}{c|}{ Área rural } & \multicolumn{2}{c|}{ Área urbana } \\
\cline { 2 - 5 } \multicolumn{1}{c|}{} & sim & não & sim & não \\
\hline a & $\underline{\mathbf{5 3 , 8}}$ & 42,3 & 36,8 & $\underline{\mathbf{6 3 , 2}}$ \\
\hline b & 46,2 & 46,2 & 50,0 & 50,0 \\
\hline c & 30,8 & $\underline{\mathbf{6 1 , 5}}$ & 26,5 & $\underline{\mathbf{7 3 , 5}}$ \\
\hline d & 15,4 & $\underline{\mathbf{7 6 , 9}}$ & 20,6 & $\underline{\mathbf{7 9 , 4}}$ \\
\hline
\end{tabular}

Assim como a saúde, o estado emocional também foi retratado, sendo que pelos escolares da área rural ele foi fator relevante para a execução das atividades, diferentemente dos escolares da área urbana (Tabela 3 ).

Tabela 3 - Dados compilados da questão 5, dividido por alternativas $(a-c)$ e áreas de estudo (rural e urbana).

\begin{tabular}{|c|c|c|c|c|}
\cline { 2 - 5 } \multicolumn{1}{c|}{} & \multicolumn{2}{c|}{ Área rural } & \multicolumn{2}{c|}{ Área urbana } \\
\cline { 2 - 5 } \multicolumn{1}{c|}{} & sim & não & sim & não \\
\hline a & 34,6 & 57,7 & 44,1 & 55,9 \\
\hline b & 46,2 & 38,5 & 42,6 & 57,4 \\
\hline c & 65,4 & 15,4 & 38,2 & 61,8 \\
\hline
\end{tabular}

Os escolares relatam que problemas emocionais na sua maioria, não afetam suas relações interpessoais, principalmente em relação a família, amigos ou grupos de pessoas (Gráfico 3). Contudo, nas últimas 4 semanas que antecederam o estudo eles apontaram sentir ou nenhuma forma de dor a dor moderada, e que na sua maioria o sentimento de dor leve, atrapalhou as atividades do trabalho ou domiciliares (Gráficos 3 e 4).

Gráfico 3 - Perfil das respostas para a pergunta 7, sendo A escolares da área rural, e B escolares da área urbana. No eixo ' $x$ ' apresenta-se as alternativas.
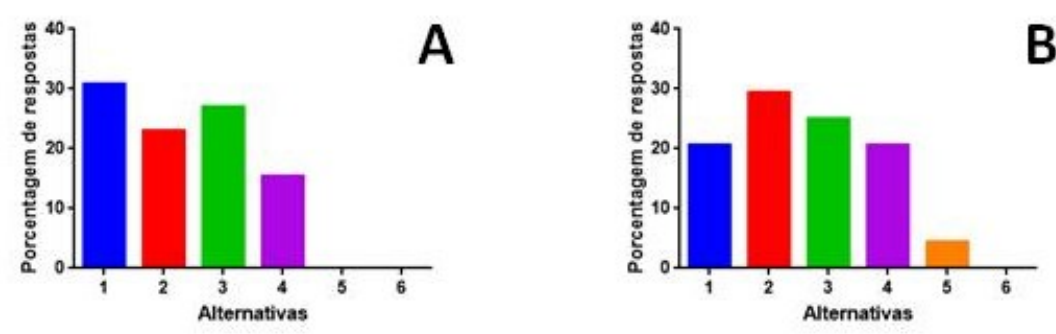

Saúde Meio Ambient. v. 6, n. 1, p. 43-53, jan./jun. 2017 
Gráfico 4 - Perfil das respostas para a pergunta 8, sendo A escolares da área rural, e B escolares da área urbana. No eixo ' $x$ ' apresenta-se as alternativas.
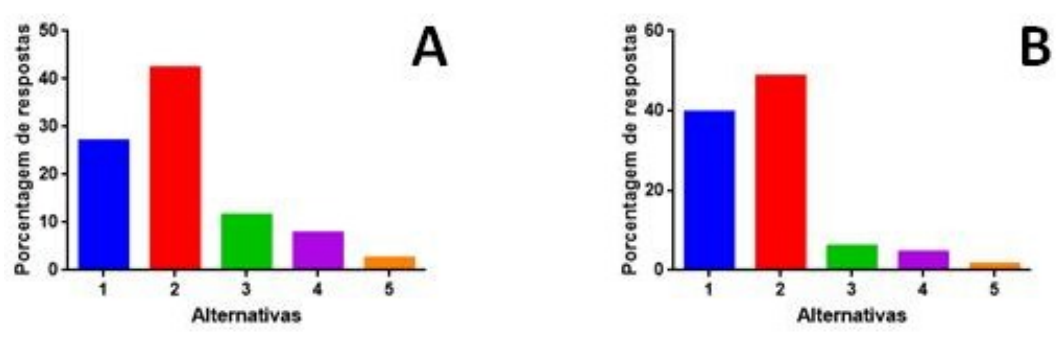

Os escolares foram indagados quanto a auto percepção de como se sentiam nas últimas 4 semanas que antecederam a avaliação, fatos como: quanto tempo eles se sentiam cheios de vigor, nervosos, deprimidos, tranqüilos, cheios de energia, desanimados, esgotados, felizes e cansados. Para os escolares da área rural, na maioria das indagações ouve a auto percepção de estarem uma boa parte do tempo com todos os sentimentos presentes, exceto para o estado de felicidade que foi retratada como permanente. Já os escolares da área urbana flutuaram em todas as alternativas passíveis, sendo que a felicidade também foi retratada como permanente, contudo, interessantemente, os mesmos relataram nunca estivessem se sentindo desanimados, deprimidos ou esgotados (Tabela 4). Ainda quanto a auto percepção, ambos os escolares (rural ou urbano) relatam que problemas físicos ou emocionais não afetam na sua grande maioria as atividades sociais, como visitar amigos e parentes, por exemplo (Gráfico 5).

Tabela 4 - Dados compilados da questão 9, dividido por alternativas $(\mathrm{a}-\mathrm{c})$ e áreas de estudo (rural e urbana).

\begin{tabular}{|c|c|c|c|c|c|c|c|c|c|c|c|c|}
\hline & \multicolumn{6}{|c|}{ Área rural } & \multicolumn{6}{|c|}{ Área urbana } \\
\hline & $\begin{array}{c}\text { Todo } \\
\text { tempo }\end{array}$ & $\begin{array}{c}\text { A maior } \\
\text { parte do } \\
\text { tempo }\end{array}$ & $\begin{array}{c}\text { Uma boa } \\
\text { parte do } \\
\text { tempo }\end{array}$ & $\begin{array}{c}\text { Alguma } \\
\text { parte do } \\
\text { tempo }\end{array}$ & $\begin{array}{c}\text { Pequena } \\
\text { parte do } \\
\text { tempo }\end{array}$ & Nuca & $\begin{array}{c}\text { Todo } \\
\text { tempo }\end{array}$ & $\begin{array}{c}\text { A maior } \\
\text { parte do } \\
\text { tempo }\end{array}$ & $\begin{array}{c}\text { Uma boa } \\
\text { parte do } \\
\text { tempo }\end{array}$ & $\begin{array}{c}\text { Alguma } \\
\text { parte do } \\
\text { tempo }\end{array}$ & $\begin{array}{c}\text { Pequena } \\
\text { parte do } \\
\text { tempo }\end{array}$ & Nuca \\
\hline$a$ & 26,9 & 11,5 & 42,3 & 7,7 & 7,7 & 0,0 & 22,1 & 19,1 & 4,4 & 11,8 & $\underline{22,1}$ & 20,6 \\
\hline b & 11,5 & 3,8 & 53,8 & 3,8 & 19,2 & 3,8 & 17,6 & 19,1 & 11,8 & 16,2 & 19,1 & 16,2 \\
\hline c & 7,7 & 7,7 & 30,8 & 11,5 & 7,7 & 30,8 & 7,4 & 17,6 & 13,2 & 5,9 & 10,3 & 45,6 \\
\hline d & 19,2 & 23,1 & 34,6 & 3,8 & 7,7 & 3,8 & 23,5 & $\underline{25,0}$ & 20,6 & 13,2 & 11,8 & 5,9 \\
\hline e & 38,5 & 15,4 & $\underline{38,5}$ & 3,8 & 3,8 & 0,0 & 23,5 & 25,0 & $\underline{27,9}$ & 11,8 & 7,4 & 4,4 \\
\hline$f$ & 11,5 & 15,4 & 23,1 & 7,7 & 30,8 & 19,2 & 2,9 & 7,4 & 17,6 & 14,7 & 22,1 & 35,3 \\
\hline g & 15,4 & 0,0 & $\underline{30,8}$ & 11,5 & 19,2 & 19,2 & 8,8 & 8,8 & 13,2 & 13,2 & 20,6 & 35,3 \\
\hline $\mathrm{h}$ & $\underline{46,2}$ & 15,4 & 30,8 & 0,0 & 0,0 & 0,0 & 39,7 & 19,1 & 8,8 & 10,3 & 13,2 & 8,8 \\
\hline $\mathbf{i}$ & 19,2 & 3,8 & 34,6 & 15,4 & 19,2 & 3,8 & 8,8 & 16,2 & 7,4 & 23,5 & 23,5 & 22,1 \\
\hline
\end{tabular}


Gráfico 5 - Perfil das respostas para a pergunta 10, sendo A escolares da área rural, e B escolares da área urbana. No eixo ' $x$ ' apresenta-se as alternativas.

A

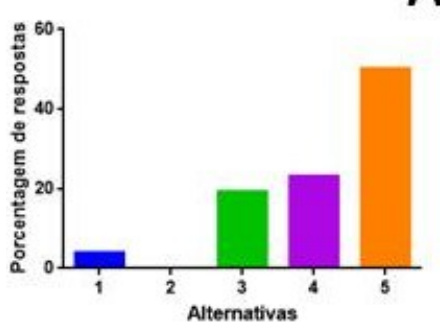

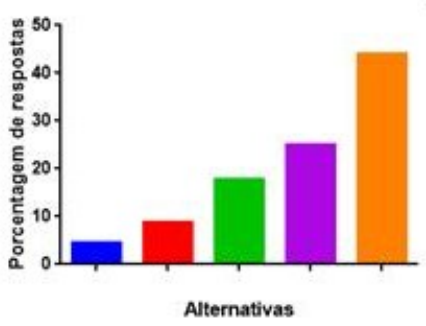

Dentro destes achados, a maioria significativa dos alunos possuem relações sociais satisfatórias em relação ao estado físico e emocional, o que já é descrito na literatura evidenciando que a presença de atividades físicas está associada diretamente com a qualidade de vida de adolescentes inclusive no contexto social, assim como, a ausência de consumo de tabaco e álcool, por exemplo (SILVEIRA et al., 2007). Outro estudo com avaliação psicomotora sobre o estilo de vida de escolares em atividades escolares e não escolares, mostrou que a presença de distúrbios psicomotores está diretamente relacionada com a preferência por atividades mais estáticas dos escolares (LUCENA et al., 2010), o qual por sua vez é de fato um interventor na qualidade de vida destes indivíduos.

Em relação ao comportamento social e comparativo, ambos os escolares (escola urbana e rural) relatam ser obedientes em relação as outras pessoas, não saber se são tão saudáveis quanto qualquer outra pessoa, ou então que seu estado de saúde possa piorar futuramente. Já a percepção do estado de saúde foi excelente para os escolares da área rural, e para os da área urbana houve o relato principalmente quanto não saberem responder (Tabela 5).

Tabela 5 - Dados compilados da questão 11, dividido por alternativas $(a-c)$ e áreas de estudo (rural e urbana).

\begin{tabular}{|c|c|c|c|c|c|c|c|c|c|c|}
\hline & \multicolumn{5}{|c|}{ Área rural } & \multicolumn{5}{|c|}{ Área urbana } \\
\hline & $\begin{array}{l}\text { Definitivamente } \\
\text { verdadeiro }\end{array}$ & $\begin{array}{c}\text { Maioria das } \\
\text { vezes } \\
\text { verdadeiro }\end{array}$ & $\begin{array}{l}\text { Não } \\
\text { Sei }\end{array}$ & $\begin{array}{c}\text { Maioria } \\
\text { das vezes } \\
\text { falso }\end{array}$ & $\begin{array}{c}\text { Definitivamente } \\
\text { falso }\end{array}$ & $\begin{array}{c}\text { Definitivamente } \\
\text { verdadeiro }\end{array}$ & $\begin{array}{c}\text { Maioria das } \\
\text { vezes } \\
\text { verdadeiro }\end{array}$ & $\begin{array}{l}\text { Não } \\
\text { Sei }\end{array}$ & $\begin{array}{c}\text { Maioria } \\
\text { das } \\
\text { vezes } \\
\text { falso } \\
\end{array}$ & $\begin{array}{c}\text { Definitivamente } \\
\text { falso }\end{array}$ \\
\hline$a$ & 34,6 & 11,5 & 34,6 & 7,7 & 7,7 & 30,9 & 25,0 & 27,9 & 5,9 & 8,8 \\
\hline b & 26,9 & 11,5 & 57,7 & 0,0 & 3,8 & 17,6 & 25,0 & 29,4 & 14,7 & 13,2 \\
\hline c & 26,9 & 3,8 & 65,4 & 0,0 & 0,0 & 7,4 & 7,4 & 52,9 & 8,8 & 23,5 \\
\hline d & 26,9 & 11,5 & 46,2 & 0,0 & 7,7 & 41,2 & 16,2 & 23,5 & 13,2 & 5,9 \\
\hline
\end{tabular}

Como pode ser visto, a discrepância pequena, mas presente, entre os ambientes escolares (urbano e rural) é um fator a ser melhor trabalhando quando se visa a melhoria da qualidade de vida destes escolares. O ministério da saúde junto com o ministério da educação tem criado programas de incentivo para a promoção à 
saúde baseado na inserção de novas rotinas de educação física no ambiente escolar (BRASIL. Ministério da Saúde, 2014), o que poderia de forma normatizada e padronizada diminuir estas diferenças. A inserção de uma educação física de qualidade no ambiente escolar tem sido o alvo em diferentes faixas etárias, e isso se deve porque esta área está dentro das medidas de atenção primária que visam a promoção à saúde, trazendo melhoras fisiológicas, comportamentais, e psicossociais. Dentro desta linha ainda, é consolidado que a condição socioeconômica dos escolares é diretamente correlacionada com o nível de atividade física ( $\mathrm{LOCH}, 2010$, p. 76).

Além dos fatores físicos, sabe-se que a rotina alimentar também possui importância na consolidação da qualidade de vida do indivíduo. O instituto brasileiro de geografia e estatística (IBGE) apresentou em 2012 dados comparativos entre a disponibilidade, e indiretamente o consumo, de produtos pouco nutritivos e com alto índice de açúcar e gordura para escolares da rede pública e particular, e achou-se que a rede pública tem grande carência ainda nesta área, sendo a rede particular uma maior incentivadora a promoção à saúde (IBGE, 2012). Contudo, sabe-se que os maus hábitos alimentares no ambiente familiar está presente com maior significância em escolares mais favorecidos no âmbito econômico, e que por conseqüência disto há nesta classe uma alta incidência de sobrepeso e obesidade (NUNES; FIGUEIROA; ALVES, 2007), o que na maioria das vezes acarreta na piora da qualidade de vida do indivíduo. Visto que este problema de excesso de peso já é uma rotina no ambiente escolar, há descrito várias propostas e incentivos para a modificação de comportamentos alimentares junto a comportamentos de atividade física associando o ambiente escolar com o ambiente familiar para melhores resultados e consolidação da proposta (NOGUEIRA; NAVEGA, 2011; BENEDET et al., 2013). Além da modificação de comportamentos alimentares e físicos há também a necessidade de atenção aos comportamentos psicossociais, pois estes são conciliados aos outros seja em uma atividade física ou na rotina alimentar.

Dentro destes achados no presente artigo, vale-se ressaltar que dependendo do perfil da qualidade de vida (seja em relação as condições físicas, sócias ou emocionais) há a necessidade de avaliações mais fidedignas e que suportem evidências clínicas para futuras intervenções (NOBRE et al., 2006). Pois sabe-se que em consequência de maus hábitos alimentares e sobrepeso e indisposição tem sido as queixas mais freqüentes entre os adolescentes, seguido de estados de desanimo e depressão. Entende-se que os dois últimos estados na sua maioria possam estar diretamente relacionados as conseqüências dos maus hábitos alimentares (NUNES; FIGUEIROA; ALVES, 2007), possivelmente através do contexto social.

\section{CONSIDERAÇÕES FINAIS}

A auto percepção da qualidade de vida por escolares adolescentes mostra um índice de bom para o estado de saúde desta respectiva população, contudo, 
alterações deste estado indicam ser responsáveis por modificações ou alterações na realização de atividades leves a moderadas. Entretanto, escolares do perímetro rural relaram maiores dificuldades quanto a conciliação de diferentes atividades. Estudos amostrais de auto percepção são cruciais para o conhecimento de indicadores populações sobre a qualidade de vida, e outros fatores. Este estudo aponta que o autoconhecimento do perfil dos escolares pode auxiliar em medidas de promoção da saúde, ou até mesmo, conhecimento da população adulta atuante das próximas décadas.

\section{REFERÊNCIAS}

BARBOSA FILHO, V. C. et al. Mudanças no estilo de vida e na percepção da saúde em estudantes do ensino médio: análise prospectiva do projeto "Saúde na Boa". Revista Brasileira de Cineantropometria e Desempenho Humano, v. 16, n. 1, p. $55-67,2014$.

BENEDET, J. et al. Excesso de peso em adolescentes: explorando potenciais fatores de risco. Revista Paulista de Pediatria, v. 31, n. 2, p.172-81, 2013.

CAVALCANTE, M. B. P. T.; ALVES, M. D. S.; BARROSO, M. G. T. Adolescência, álcool e drogas: uma revisão na perspectiva da promoção da saúde. Escola Anna Nery Revista de Enfermagem, v. 12, n. 3, p. 555-59, 2008.

CICONELLI, R. M. et al. Tradução para a língua portuguesa e validação do questionário genérico de avaliação de qualidade de vida SF-36 (Brasil SF-36).

Revista Brasileira de Reumatologia, v. 38, n. 3, p. 143-150, 1999.

FARIAS JÚNIOR, J. C. et al. Comportamento de risco à saúde em adolescentes no sul do Brasil: Prevalência e fatores associados. Revista Panamericana de Salud Publica, v. 25, n. 4, p. 344-352, 2009.

GIL, A. C. Métodos e técnicas de pesquisa social. São Paulo: Atlas, 1999.

INSTITUTO BRASILEIRO DE GEOGRAFIA E ESTATÍSTICA. Pesquisa Nacional de Saúde do escolar. 2012.

LOCH, M. R. A promoção da atividade física na escola: um difícil e necessário desafio. Revista Brasileira de Atividade Física e Saúde, v. 16, n. 1, p. 76-77, 2011.

LUCENA, N. M. G. et al. Lateralidade manual, ocular, e dos membros inferiores e sua relação com déficit de organização espacial em escolares. Estudos de Psicologia, v. 27, n. 1, p. 3-11, 2010.

KERLINGER, F. N. Metodologia da pesquisa em ciências sócias: um tratamento conceitual. São Paulo: EPU, 1980. 
KUNKEL, N.; OLIVEIRA, W. F.; PERES, M. A. Excesso de peso e qualidade de vida relacionado à saúde em adolescentes em Florianópolis, SC. Revista de Saúde Pública, v. 43, n. 2, p. 226-225, 2009.

NOBRE, M. R. C. et al. Prevalências de sobrepeso, obesidade e hábitos de vidas associados ao risco cardiovascular em alunos do ensino médio. Revista da Associação Médica Brasileira, v. 52, n. 2, p. 118 -224, 2006.

NOGUEIRA, H. C.; NAVEGA, M. T. Influencia da escola de postura na qualidade de vida, capacidade funcional, intensidade de dor e flexibilidade de trabalhos administrativos. Fisioterapia e pesquisa, v. 18, n. 4, p. $353-358,2011$.

NUNES, M. M. A.; FIGUEIROA, J. N.; ALVES, J. G. B. Excesso de peso, atividade física, e hábitos alimentares de diferentes classes econômicas de Campina Grande (PB). Revista Associação Médica Brasileira, v. 53, .n. 2, p. 130-4, 2007.

QUINTAS, J. S. Educação no processo de gestão ambiental: uma proposta de educação ambiental transformadora e emancipatória. Brasília: Ministério do Meio Ambiente: Identidade da Educação Ambiental Brasileira. 2004.

RAYMUNDO, L. C.; KUHNEN, A. A psicologia e a educação ambiental. Revista de Ciências Humanas, v. 44, n. 2, p. 435-450, 2010.

SILVEIRA, M. F. et al. Qualidade de vida entre adolescentes: estudo seccional empregando o SF 12, Ciência e Saúde Coletiva, v. 18, n. 7, p. 2007-2015, 2007.

VINGILIS, E. R.; WADE, T. J.; SEELEY, J. S. Predictors of Adolescent Self-Rated Health Analysis of the National Population Health Survey. Can J Public Health, v. 93, n. 3, p. 193-197, 2002.

TENÓRIO, M. C. M. et al. Atividade física e comportamento sedentário em adolescentes estudantes do ensino médio. Revista Brasileira de Epidemiologia, v. 13 , n. 1, p. 105-17, 2010.

The WHOQOL Group. The World Health Organization quality of life assessment (WHOQOL): position paper from the World Health Organization. Soc Sci Med, v. 41, n. 10, p. 1403-1409, 1995.

Artigo recebido em: 26/05/2015

Artigo aprovado em: 22/06/2017 